\title{
Quantum Entanglement in Electric Circuits: from Anomalous Crosstalk to Electromagnetic Compatibility in Nano-Electronics
}

\author{
Gregory Slepyan, Amir Boag \\ School of Electrical Engineering \\ Tel-Aviv University \\ Tel-Aviv, Israel \\ Giovanni Miano \\ Dept. of Electr. Engineering and Information Technology \\ University of Naples Federico II \\ Naples, Italy \\ Sergey Maksimenko, Polina Kuzhir \\ Research Institute for Nuclear Problems \\ Belarusian State University \\ Minsk, Belarus
}

\begin{abstract}
We show that the electromagnetic coupling at the nanoscale may be accompanied by another coupling mechanism, related to quantum entanglement. Consequently, a combined "electromagnetic-quantum" coupling is created, which stipulates long-distance and long-living interactions in electric circuits. Manifestation of this effect in electromagnetic compatibility (EMC) is discussed. An efficient theoretical framework for EMC analysis in nanoelectronics is developed based on the generalized theory of electric circuits. It is shown that the action of quantum entanglement is equivalent to an addition of the supplementary elements in electric circuit with the effective admittances defined as general susceptibilities that can be calculated using the Kubotechnique.
\end{abstract}

Keywords - Electromagnetic crosstalk; quantum entanglement; quantum dots; nano-antennas; nano-EMC

\section{INTRODUCTION}

The idea of quantum entanglement appears in two famous paradoxes of quantum theory (Shrödinger's cat paradox [1] and paradox of Einstein-Podolsky-Rosen [2]). For many years entanglement was of special interest in the context of experimental studies aimed at proving the completeness of quantum mechanics. The present-day developments in nanotechnologies make the entanglement phenomena relevant for the proper operation of nanoelectronic devices and systems. Recently, quantum entanglement was identified to be a promising tool facilitating the growth in the level of integration and the reduction of the operation power in nanoelectronics and nanooptics. Among the promising devices based on the entanglement, are quantum qubits of various types [3] and quantum antennas [4,5].

This work was supported in part by EU grants FP7-PEOPLE-2009-IRSES247007 CACOMEL and FP7-PEOPLE-2013-IRSES- 612285 CANTOR.

\author{
Vladimir Mordachev, Eugene Sinkevich \\ EMC R\&D Laboratory \\ Belarusian State Univ. of Informatics and Radioelectronics \\ Minsk, Belarus \\ Mikhail E. Portnoi \\ School of Physics \\ University of Exeter \\ Exeter, UK \\ Antonio Maffucci \\ Department of Electrical and Information Engineering \\ University of Cassino and Southern Lazio \\ Cassino, Italy
}

The progress in nanoelectronics is accompanied by the general tendency towards enhancing the role of the dense and highly integrated environment in the behavior of individual nanodevices. Similar problems, associated with Electromagnetic Compatibility (EMC), has been known since the early days of radio and telegraph communications. They are associated with the ability of electromagnetic field to cause interference in electrical and electronic devices. The crosstalk - a mutual interaction among neighboring communication channels via the near field penetration from one to another - is one of the most notable manifestations of such interference [6].

Being a mechanism of quantum interference [7], entanglement is able to produce a combined "electromagnetic - quantum" interference, which we name "quantum crosstalk". Consequently, in view of the quantum crosstalk the classical EMC concepts like coupling, shielding, and matching, were reconsidered with respect to nanodevices [8-11].

In this article, we consider the basis concept, which opens the efficient way of control and suppression of the two crosstalk components in nanoelectronic design.

\section{QUANTUM ENTANGLEMENT IN ELECTRIC CIRCUITS}

Let us consider the system depicted in Fig. 1. Two quantum emitters are capacitively coupled to the correspondent pair of electrodes through which they are connected to the circuit. Two quantum dots (QDs) are coupled via two mechanisms: quantum entanglement dictated by the dipole-dipole (d-d) interaction, and capacitive electric coupling. The physical origin of $\mathrm{d}-\mathrm{d}$ interaction is via the inter-atomic exchange by the virtual photon over the common photonic bath. For brevity, we refer to the quantum object as an "atom" 
regardless of its physical nature, e.g., QD, polar molecule, etc.

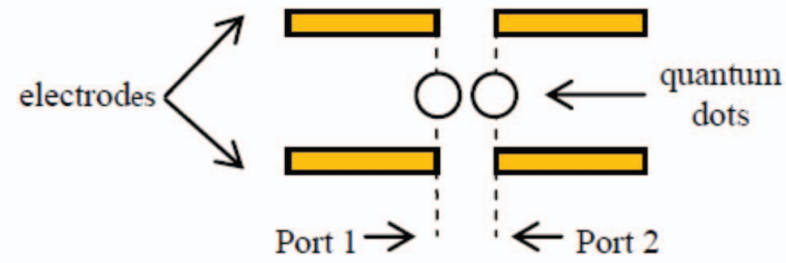

Fig.1. Pair of dipole-dipole (d-d) coupled atoms in the different electric circuits.

The two atoms interact with a classical monochromatic electromagnetic field $\mathbf{E}=\operatorname{Re}\left\{\mathbf{E}_{0} \exp (-i \omega t)\right\}$. This system is described by an effective Hamiltonian

$$
\hat{H}=\hat{H}_{a}+\hat{H}_{d d}+\hat{H}_{\text {int }} \text {, }
$$

where $\hat{H}_{a}=\hbar\left(\omega_{0}-i \gamma / 2\right) \sum_{j=1,2} \hat{\sigma}_{j z} / 2$ is the atomic component with $\hat{\sigma}_{j z}$ being inversion Pauli matrix for $j$-th atom and $\gamma$ - the decay rate of the atomic transition. The second term $\hat{H}_{d d}=\hbar\left(\Omega-i \gamma_{12} / 2\right)\left(\hat{\sigma}_{1}^{+} \hat{\sigma}_{2}^{-}+\right.$H.c. $)$is the interatomic d-d component, where $\Omega$ is the collective Lamb shift, $\gamma_{12}$ is responsible for cooperative radiative decay, $\hat{\sigma}_{j}^{ \pm}$ are the creation-annihilation operators for the excited state in the $j$-th atom. Also in (1), the last term $\hat{H}_{\mathrm{int}}=-e \sum_{j=1,2}(\vec{\mu} \cdot \mathbf{E})\left(\hat{\sigma}_{j}^{+}+\hat{\sigma}_{j}^{-}\right) \quad$ is the atom-field interaction component, where $\mathbf{E}_{j}$ is the field value at the location of the $j$-th atom. Two first components of the effective Hamiltonian are non-Hermitian due to the presence of radiative losses.

Let us assume that the system interacts with the external field in the regime of weak coupling (the term $\hat{H}_{\text {int }}$ should be considered as a small perturbation). Weak coupling (linear response) theory is based on the correct set of zero-order states, transformed due to the d-d interactions (eigenstates of Hamiltonian $\left.\hat{H}=\hat{H}_{a}+\hat{H}_{d d}\right)$. The "singly excited states" due to the entanglement and energy splitting are transformed into Dicke states:

$$
\begin{aligned}
& \left|\psi_{+}\right\rangle=\frac{1}{\sqrt{2}}(|e g\rangle+|g e\rangle), \\
& \left|\psi_{-}\right\rangle=\frac{1}{\sqrt{2}}(|e g\rangle-|g e\rangle),
\end{aligned}
$$

which, appearing in addition to the ground $|g g\rangle$ and doubly-

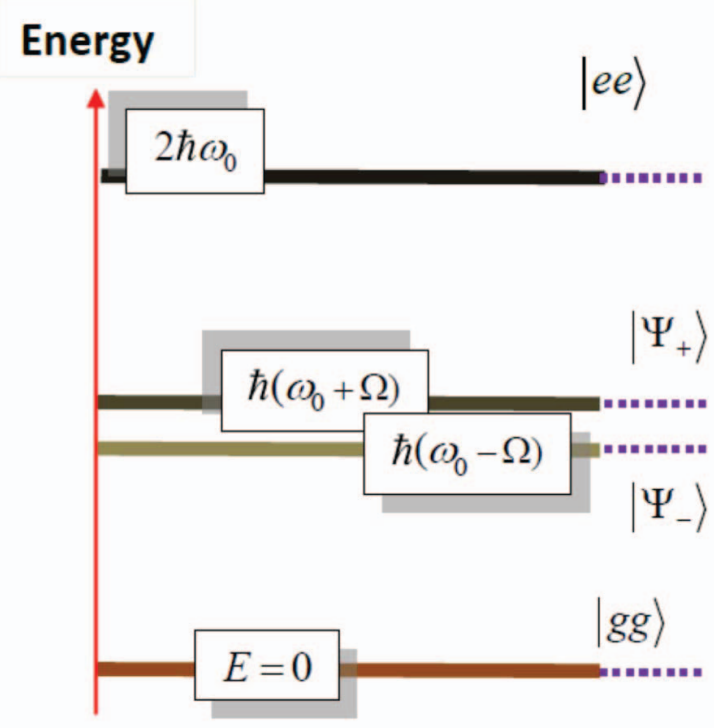

Fig.2. Four-level energy spectrum of the system under consideration.

excited $|e e\rangle$ states, form the four-level energy spectrum presented in Fig. 2.

State $\left|\psi_{+}\right\rangle$in (2) corresponds to the wavefunction that is symmetric with respect to the transposition of the atoms, and is referred to as the "superradiant" state. State $\left|\psi_{-}\right\rangle$in (3) denotes the case of antisymmetric wavefunction and it is named the "subradiant" state [5]. Superradiant and subradiant states are different in terms of their resonance frequencies $\omega_{ \pm}=\omega_{0} \pm \Omega$ and decay rates $\gamma_{ \pm}=\gamma \pm \gamma_{12}$.

The equivalent circuit for this case is obtained based on the concept of generalized susceptibility (Kubo-approach) [12] and has a form of a two-port network. To examine the role of the quantum correlation in the crosstalk phenomenon, we have to take into account also the capacitive coupling between two circuits. To that end, we represent the overall system, i.e., the two coupled quantum emitters together with the two electrode pairs, through the equivalent $\pi$-type twoport depicted in Fig. 3. The contributions of the electrodes are taken into account through the capacitance $C_{e g}$ at each port, and by an inter-circuit capacitance, $C_{L R}$, This capacitance consists of two components: the coupling between the left (L) and right $(\mathrm{R})$ electrodes and the coupling between $\mathrm{L}(\mathrm{R})$ atom with $\mathrm{R}(\mathrm{L})$ electrode. Then, using classical synthesis formulas for a $\pi$-type two-port, it is easy to compute the element admittances in Fig. 3. 


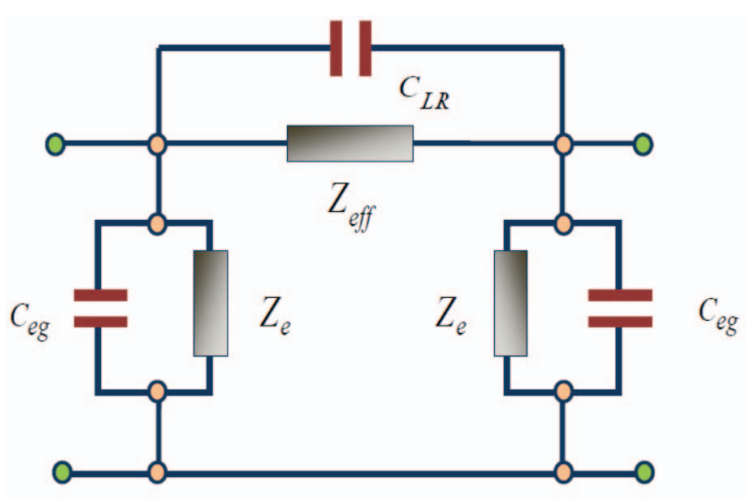

Fig. 3. Equivalent electric circuit of the two atoms coupled via dipole-dipole (d-d) interaction. $Z_{\text {eff }}(\omega)$ is the additional element, produced due to the quantum entanglement.

The impedances $Z_{e}(\omega)$ and $Z_{\text {eff }}(\omega)$ are given by

$$
\begin{aligned}
& \frac{1}{Z_{e}(\omega)}=\frac{1}{2}\left[\frac{1}{Z_{e}^{+}(\omega)}+\frac{1}{Z_{e}^{-}(\omega)}\right] \\
& \frac{1}{Z_{e f f}(\omega)}=\frac{1}{2}\left[\frac{1}{Z_{e}^{+}(\omega)}-\frac{1}{Z_{e}^{-}(\omega)}\right]
\end{aligned}
$$

where

$$
Z_{e}^{ \pm}(\omega)=i \hbar \frac{\omega_{ \pm}^{2}-\omega^{2}-i \omega \gamma_{ \pm}}{2 \mu^{2} \omega \omega_{ \pm}} A_{e f f},
$$

$\mu=|\vec{\mu}|$ is the dipole moment of the transition, and $A_{\text {eff }}$ is the effective area of quantum confinement.

Only the super-radiant mode contributes to the impedance $Z_{e}(\omega)$, while $Z_{\text {eff }}(\omega)$ depends on both the super-radiant and sub-radiant modes. Two different mechanisms contribute to the inter-circuit coupling: the capacitive coupling associated in Fig. 3 with the capacitance $C_{L R}$ and the quantum correlation between the two atoms, represented by the term $Z_{\text {eff }}(\omega)$. So, we obtained the fundamental result of our study: the action of quantum entanglement is equivalent to an additional element in electric circuit with the impedance given by (5). As follows from (5), the value of the resistance of this element in some frequency range is negative. Following Schrödinger [1], it doesn't contradict the thermodynamic equilibrium, because the whole system can be less uncertain than either of its entangled parts. This means, that the whole equivalent circuit is better specified than its elements. Thus, the negative resistance of a circuit element means that there is a special type of energy transfer inside the system, not an energy supply from outside. The appearance of negative resistance strongly contradicts the intuitive concepts of classic crosstalk, where the coupling channel of electromagnetic nature should be described by passive elements only [6].

The Lamb shift depends on the distance between the two atoms and decreases as the distance increases. Nevertheless, the above behavior of the crosstalk voltage only depends on the key requirement $\Omega \gg>\gamma_{ \pm}$, hence we may have a strong crosstalk even for large interatomic distances. On the other hand, in the classical crosstalk mechanism (if we disregard the quantum correlation contribution to the crosstalk), the electromagnetic field penetration from one port to the other does not couple the atomic modes and strongly decreases with the interatomic distance.

It was shown that the combination of entanglement and electromagnetic interactions is able to dramatically change the physical picture of the crosstalk. For small values of the collective Lamb-shift the impedances $Z_{e}^{ \pm}$are comparable, thus the amplitude of the coupling admittance $Z_{\text {eff }}(\omega)$ is small and the quantum correlation contribution to the crosstalk is negligible. In the limit of zero Lamb-shift, the impedances $Z_{e}^{+}$and $Z_{e}^{-}$become equal and, as a result, the quantum correlation contribution to the crosstalk completely vanishes. If $\Omega \gg>\gamma_{ \pm}$, the impedances $Z_{e}^{+}$and $Z_{e}^{-}$are substantially different at the given frequency, and consequently, the super-radiant and sub-radiant spectral lines are splitted. Thus, in contrast with classical crosstalk, the excitation produced in one of the ports may be redistributed in equal parts between the two ports due to the quantum correlation, in spite of the rather small inter-atomic interaction.

\section{FROM TODAY TO TOMORROW: ELECTROMAGNETIC COMPATIBILITY ON THE NANO-SCALE}

Various types of nanodevices are composed of nanowires connecting either conventional loads or quantum elements in entangled states. The circuits containing nanoscale components have a very high level of integration on the order of $10^{7}-10^{8}$ elements [8]. The presence of many parasitic cross-links, the number of which exceeds the number of system elements, is a major challenge for realistic noise estimates. Some of such challenging problems are related to the computational burden in electromagnetic analysis. Others are dictated by the inter-element interactions of nonelectromagnetic origin via quantum entanglement and have no analogs in the classical EMC. An example of such coupling via $\mathrm{d}$-d interaction has been presented above. In general, such coupling mechanisms are based on other types of interactions (for example, phononic, nuclear spin, electron spin, etc.,) and their combinations.

The normal operation of many nanodevices (in particular, digital elements) is defined by the ratio of the decoherence time and the time for which a system remains quantummechanically coherent. These two times are both determined by the strength of the coupling of the system to the external 


\section{ICSEE International Conference on the Science of Electrical Engineering}

world. For different systems this currently achievable ratio varies over a wide range (from $10^{4}$ to $10^{14}$ ) [3]. It is important to note that the novel mechanism of crosstalk considered above is relevant as long as the system is coherent. Therefore, its contribution is important for the whole duration of a device operation even when this duration is quite short.

Nano-EMC modeling must consider self-consistently Maxwell's equations and the quantum many-body problems. Moving from the macroscopic to the atomic scale, one can note different approaches to the quantum modeling of electron transport. We propose, as an efficient theoretical framework for nano-EMC analysis, the theory of electric circuits with quantum emitters (implemented on the cold atoms, trapped ions, quantum dots, etc.). Towards bridging the classical electric circuit theory with the quantum theory of nanoobjects, the atoms inside nano-devices are described by their effective admittances. The effective admittance is defined as a general susceptibility and calculated using the Kubotechnique [12].

This effective admittances belong to the class of Onsager kinetic coefficients, which obey some general symmetry rules [12]. This helps to identify the range of effects that are important for nano-EMC.

In particular, such effects can open the ways for the quantum crosstalk suppression. The classical tools of suppression (such as electric shunting, screening, and attenuation) are not easy to implement on the nano-scale, thus a special means based on the quantum effects become potentially important. One of such mechanisms is based on the use of the additional atomic energy levels. A promising example involving tunneling over the dark energy levels is proposed in [11]: it was shown that its action is equivalent to the effective electrical shunting of the coupling impedance.

Our work suggests a number of follow-up studies aiming to: (i) extend our consideration for other mechanisms of interatomic coupling and quantum entanglement (tunneling, spin-spin interactions, dissipative coupling via the common reservoir [13], etc); (ii) investigate the equivalent circuits for multi-level and initially pumped quantum structures; (iii) account for decoherence using the theory of open quantum systems [14]; (vi) account the influence of electrostatic, magnetostatic fields and their combinations.

\section{REFERENCES}

[1] E. Schrödinger, "Die gegenwaertige Situation in der Quantenmechanik.“ Naturwissenschaften, vol. 23, pp. 823-828, 1935.

[2] A. Einstein, B. Podolsky, and N. Rosen, "Can Quantum-Mechanical Description of Physical Reality be Considered Complete?”, Phys. Rev. vol. 47, p. 777, 1935.

[3] M. Nielsen, and I. Chuang, Quantum computation and quantum information, Cambridge University Press, Cambridge, 2012.

[4] G. Y. Slepyan, and A. Boag, "Quantum Nonreciprocity of Nanoscale Antenna Arrays in Timed Dicke States,” Phys. Rev. Lett. vol. 111, 023602 , July 2013.

[5] S. Mokhlespour, J. E. M. Haverkort, G. Slepyan, S. Maksimenko and A. Hoffmann, "Collective spontaneous emission in coupled quantum dots: Physical mechanism of quantum nanoantenna", Phys. Rev. vol. B 86, 245322, Dec. 2012.
[6] H. W. Ott, Electromagnetic Compatibility Engineering, John Wiley \& Sons, 2009.

[7] M. O. Scully and M. S. Zubairy, Quantum Optics, Cambridge University Press, Cambridge, 2001.

[8] G. Slepyan et al., "Electromagnetic Compatibility Concepts at Nanoscale", Proc. of IEEE Intern. Conf, on EMC, EMC 14, paper \# 13A1-H4, Tokyo, Japan, 2014.

[9] G. Slepyan et. al, "Equivalent Electrical Multiport for Quantum Systems in Entangled States", Proc. of IEEE Intern. Conf. on Nanotechnology, IEEE NANO-2015, Rome, Italy, paper no. 420, July 2015.

[10] G. Slepyan et al., "Nanoscale Electromagnetic Compatibility: Quantum Coupling and Matching in Nanocircuits", IEEE Trans. on Electromagnetic Compatibility, vol. 57, pp. 1645-1654, Dec. 2015.

[11] G. Slepyan et al. "Electromagnetic Compatibility in Nano-Electronics: Manifestation and Suppression of Quantum Crosstalk", IEEE COMCAS-2015 International Conference on Microwaves, Communications, Antennas and Electronic Systems, Tel Aviv, Israel Nov. 2015.

[12] L. D. Landau and E. M. Lifshitz, Statistical Physics, Course of Theoretical Physics, 3rd ed., vol. 5, Pergamon Press, 1980.

[13] D. Mogilevtsev, G. Y. Slepyan, E. Garusov, S. Y. Kilin, and N. Korolkova, "Quantum tight-binding chains with dissipative coupling", New J. Phys. vol. 17, 043065, Apr. 2015.

[14] H.-P. Breuer and F. Petruccione, The theory of open quantum systems, Clarendon Press, Oxford, 2002. 